\title{
Global classical solutions to the 3D Nordström-Vlasov system
}

\author{
Simone Calogero \\ Institutt for matematiske fag \\ NTNU Alfred Getz' vei 1 N-7491 \\ Trondheim, Norway \\ E-mail: simone.calogero@math.ntnu.no
}

\begin{abstract}
The Nordström-Vlasov system describes the evolution of selfgravitating collisionless matter in the framework of a relativistic scalar theory of gravitation. We prove global existence and uniqueness of classical solutions for the corresponding initial value problem in three dimensions when the initial data for the scalar field are smooth and the initial particle density is smooth with compact support.
\end{abstract}

\section{Introduction}

This paper is concerned with the Cauchy problem for the Nordström-Vlasov system. The latter is a Lorentz invariant kinetic model describing the evolution of self-gravitating collisionless matter under the assumption that the gravitational forces are mediated by a scalar field. In a system of Cartesian coordinates $(t, x), t \in \mathbb{R}, x \in \mathbb{R}^{3}$, the Nordström-Vlasov system is given by

$$
\begin{gathered}
\partial_{t}^{2} \phi-\Delta \phi=-\mu, \\
\mu(t, x)=\int f(t, x, p) \frac{d p}{\sqrt{1+|p|^{2}}}, \\
S f-\left[(S \phi) p+\left(1+|p|^{2}\right)^{-1 / 2} \nabla_{x} \phi\right] \cdot \nabla_{p} f=4 f S \phi .
\end{gathered}
$$

Here $p \in \mathbb{R}^{3}$ is the momentum variable, $f=f(t, x, p)$ is the particle density in phase-space, $\phi=\phi(t, x)$ is the scalar gravitational field generated by the particles and

$$
S=\partial_{t}+\widehat{p} \cdot \nabla_{x}, \quad \widehat{p}=\frac{p}{\sqrt{1+|p|^{2}}} ;
$$

$S$ is the free-transport operator, $\widehat{p}$ denotes the relativistic velocity of a particle with momentum $p$. Units are chosen such that the mass of each particle, the 
gravitational constant and the speed of light are equal to unity. A solution $(f, \phi)$ of this system is interpreted as follows: The space-time is a four-dimensional Lorentzian manifold with a conformally flat metric which, in the coordinates $(t, x)$, takes the form

$$
g_{\mu \nu}=e^{2 \phi} \operatorname{diag}(-1,1,1,1), \mu, \nu=0, \ldots 3 .
$$

The particle density on the mass shell in this metric is $\mathfrak{f}=e^{-4 \phi} f\left(t, x, e^{\phi} p\right)$, but it is more convenient to work with $f$ and $\phi$ as the dynamic variables. The Vlasov equation (1.3) is equivalent to the condition that $\mathfrak{f}$ is constant along the geodesics flow of the metric (1.4). The right hand side of the field equation (1.1) is the trace of the stress energy tensor associated to $f$ with respect to the background Minkowski metric. We refer to 4 for a derivation of the equations.

Although scalar theories of gravity are not physically correct, they may provide useful simplified models for General Relativity [25]. Moreover, scalar fields play a central role in modern theories of classical and quantum gravity [8]. The physically correct relativistic model for self-gravitating collisionless matter is the Einstein-Vlasov system, which is discussed for instance in [1, 21]. Due the very complicated structure of the Einstein equations, the evolution problem for the Einstein-Vlasov system remains poorly understood, even in spherical symmetry. In fact, global existence and uniqueness of (asymptotically flat) solutions to the Einstein-Vlasov system has been proved only for small data in spherical symmetry [21]. As opposed to this, the Cauchy problem for the Vlasov-Poisson system, which is the non-relativistic limit of the Einstein-Vlasov system [22, 23, is by now well-understood, cf. [16, 18, 20, 24. In [5] it is shown that Vlasov-Poisson is the non-relativistic limit of the Nordström-Vlasov system as well. However, global existence of classical solutions for the Nordström-Vlasov system and related models - such as the Vlasov-Maxwell system of plasma physics - has not yet been established. In this paper we show that this fundamental question has a positive answer for the Nordström-Vlasov system. Precisely, we shall prove the following

Theorem 1 Given $f_{0}: \mathbb{R}^{3} \times \mathbb{R}^{3} \rightarrow[0, \infty)$ and $\phi_{0}, \phi_{1}: \mathbb{R}^{3} \rightarrow \mathbb{R}$ satisfying

$$
f_{0} \in C_{c}^{1}, \phi_{0} \in C_{b}^{3} \cap H^{1}, \phi_{1} \in C_{b}^{2} \cap L^{2},
$$

there exists a unique global solution $(f, \phi)$ of the Nordström-Vlasov system in the class

$$
(f, \phi) \in C^{1}\left([0, \infty) \times \mathbb{R}^{3} \times \mathbb{R}^{3}\right) \times C^{2}\left([0, \infty) \times \mathbb{R}^{3}\right),
$$

such that $(f, \phi)_{\mid t=0}=\left(f_{0}, \phi_{0}\right)$ and $\left(\partial_{t} \phi\right)_{\mid t=0}=\phi_{1}$.

The proof of Theorem 1 relies upon three main tools. A first one is the representation formula for the time derivative of the field given in [6 Prop. 1]. It turns out that estimating $\partial_{t} \phi$ is enough to obtain global existence. A second important tool is given by the (null cone) energy estimates which derive from the energy identity

$$
\partial_{t} e+\nabla_{x} \cdot \mathfrak{p}=0
$$


where

$$
e(t, x)=\int \sqrt{1+|p|^{2}} f d p+\frac{1}{2}\left(\partial_{t} \phi\right)^{2}+\frac{1}{2}\left(\nabla_{x} \phi\right)^{2}, \quad \mathfrak{p}(t, x)=\int p f d p-\partial_{t} \phi \nabla_{x} \phi .
$$

Upon integration, 1.5 leads to the identities

$$
\begin{gathered}
\iint \sqrt{1+|p|^{2}} f d p d x+\int\left[\frac{1}{2}\left(\partial_{t} \phi\right)^{2}+\frac{1}{2}\left(\nabla_{x} \phi\right)^{2}\right] d x=\text { const. } \\
\int_{|x-y| \leqslant t}(e+\mathfrak{p} \cdot \omega)(t-|x-y|, y) d y=\int_{|x-y|=t} e(0, y) d S_{y}, \quad \omega=\frac{(y-x)}{|x-y|} .
\end{gathered}
$$

Note also that

$$
e+\mathfrak{p} \cdot \omega=\int\left(\sqrt{1+|p|^{2}}+\omega \cdot p\right) f d p+\frac{1}{2}\left(\omega \wedge \nabla_{x} \phi\right)^{2}+\frac{1}{2}\left(\partial_{t} \phi-\omega \cdot \nabla_{x} \phi\right)^{2} .
$$

We shall refer to (1.7) as the null cone energy identity, while (1.6) is the usual conservation of total energy. They imply

$$
\begin{gathered}
\left\|\partial_{t} \phi(t)\right\|_{L^{2}\left(\mathbb{R}^{3}\right)}+\left\|\nabla_{x} \phi(t)\right\|_{L^{2}\left(\mathbb{R}^{3}\right)} \leqslant \text { const. } \\
\left\|\left(\omega \wedge \nabla_{x} \phi\right)(t, x)\right\|_{L^{2}\left(\Lambda_{t, x}\right)}+\left\|\left(\partial_{t} \phi-\omega \cdot \nabla_{x} \phi\right)(t, x)\right\|_{L^{2}\left(\Lambda_{t, x}\right)} \leqslant \text { const. } \times t^{2},
\end{gathered}
$$

where $\mathbb{R}^{4} \supset \Lambda_{t, x}=\{(t-|x-y|, y):|x-y| \leqslant t\}_{y \in \mathbb{R}^{3}}$ is the past light cone with vertex at $(t, x)$ and base on $t=0$. The energy estimates are used to bound $\partial_{t} \phi$. For this purpose the representation formula for $\partial_{t} \phi$ must be rewritten in a proper way to single out the contributions which are bounded by the null cone energy. All terms in the integral representation of $\partial_{t} \phi$ but one can be estimated using the energy estimates. The remaining term is estimated using the third-and most important - main ingredient in the proof of Theorem 11 which is the $L^{2}$ version of a lemma due to C. Pallard, see [17. Lemma 1.1]. This crucial lemma establishes an $L^{\infty}$ bound for the time integral of functions evaluated along the characteristics of the Vlasov equation. As we shall need a slightly different formulation of the result proved in [17], a proof thereof will be given when it is needed.

We remark that prior to the present result, global existence theorems for Nordström-Vlasov have been proved under certain restrictions, such us small data [10], spherical symmetry [2] and for the 2-dimensional system 15]. Global existence of weak solutions is established in [7. The proofs of these results make use of techniques originally developed to study the Cauchy problem for the Vlasov-Maxwell system, see 3, 9, 11, 12, 13, 14, 19. This suggests that if the analogue of Theorem [1 hold for the Vlasov-Maxwell system, the proof thereof might rely upon similar arguments as to the ones presented in this paper. However, since for Vlasov-Maxwell one has to estimate a vector field instead of a scalar field, the proof of global existence in the plasma physics case might be considerably more difficult and require some additional non-trivial idea. 


\section{Preliminaries}

Throughout the paper we denote by $C(t)$ any continuous non-decreasing positive function of time. If it is a constant, we denote it simply by $C$. The characteristics of the differential operator in the left hand side of (1.3) are the solutions of

$$
\dot{x}=\widehat{p}, \quad \dot{p}=-(S \phi) p-\left(1+|p|^{2}\right)^{-1 / 2} \nabla_{x} \phi
$$

and we denote by $(X, P)(s)$ the characteristic curve satisfying $(X, P)(t)=(x, p)$. Note that $(X, P)(s)$ also depends on $(x, p)$, but this is not reflected in our notation. The function $e^{-4 \phi} f$ is constant along the solutions of 2.10). We deduce that

$$
f(t, x, p)=f_{0}(X(0), P(0)) \exp \left[4 \phi(t, x)-4 \phi_{0}(X(0))\right],
$$

whence

$$
\left\|e^{-4 \phi} f(t)\right\|_{\infty} \leqslant C .
$$

Let $\phi=\phi_{\text {hom }}+\psi$, where $\psi$ is the solution of (1.1) with zero data and $\phi_{\text {hom }}$ solves $\square \phi=0$ with data $\left(\phi_{0}, \phi_{1}\right)$. Since $f \geqslant 0$, then $\psi \leqslant 0$. Therefore

$$
\phi(t, x) \leqslant C(t),
$$

and

$$
e^{\phi}|\phi| \leqslant C(t)
$$

For (2.14) we used that $\sup _{\xi \leqslant 0}\left(e^{\xi}|\xi|\right) \leqslant C$. Note also that $\|f(t)\|_{\infty} \leqslant C(t)$. In [6] it is proved that the Cauchy problem for the Nordström-Vlasov system has a unique classical solution locally in time. Let $T_{\max }$ be the maximal time of existence and denote

$$
\tilde{\mathcal{P}}(t)=\sup _{0 \leqslant s<t}\left\{|p|: f(s, x, p) \neq 0, \text { for some } x \in \mathbb{R}^{3}\right\} .
$$

In 66. 7] it is proved that $\tilde{\mathcal{P}}\left(T_{\max }\right)<\infty \Rightarrow T_{\max }=\infty$, i.e., the solution could blow-up in finite time only if the momentum support of $f$ becomes unbounded. However, for the proof of Theorem 1 it is essential to look at another quantity. Define

$$
\mathcal{P}(t)=\sup _{0 \leqslant s<t}\left\{e^{\phi} \sqrt{1+|p|^{2}}: f(s, x, p) \neq 0, \text { for some } x \in \mathbb{R}^{3}\right\},
$$

the maximal particles energy in the support of $f$.

Lemma 1 The assertions $\tilde{\mathcal{P}}(t)<\infty$ and $\mathcal{P}(t)<\infty$ are equivalent. In particular

$$
\mathcal{P}\left(T_{\max }\right)<\infty \Rightarrow T_{\max }=\infty .
$$

Proof: Since $e^{\phi} \leqslant C(t)$, then we have $\mathcal{P}(t) \leqslant C(t) \sqrt{1+\tilde{\mathcal{P}}(t)^{2}}$. Moreover, using (2.12)- (2.13),

$$
\mu(t, x) \leqslant \int_{|p| \leqslant e^{-\phi} \mathcal{P}(t)} f \frac{d p}{\sqrt{1+|p|^{2}}} \leqslant C e^{2 \phi} \mathcal{P}(t)^{2} \leqslant C(t) \mathcal{P}(t)^{2} ;
$$


hence $\mathcal{P}(t)<\infty$ implies $\|\mu(t)\|_{\infty} \leqslant C(t)$ and therefore also $\|\phi(t)\|_{\infty} \leqslant C(t)$. Thus

$$
\mathcal{P}(t)<\infty \Rightarrow \tilde{\mathcal{P}}(t) \leqslant\left(\sup _{s \in[0, t)} e^{\|\phi(s)\|_{\infty}}\right) \mathcal{P}(t) \leqslant C(t) .
$$

In order to estimate the function $\mathcal{P}(t)$ we shall use that, along characteristics,

$$
\frac{d}{d s} e^{2 \phi}\left(1+|p|^{2}\right)=2 e^{2 \phi} \partial_{s} \phi
$$

The aim is to transform (2.16) in a Grönwall's type inequality by estimating $\partial_{s} \phi$ in terms of $\mathcal{P}(t)$. An estimate like $\left|\partial_{t} \phi\right| \leqslant C(t) \mathcal{P}(t)^{2} \log \mathcal{P}(t)$ would be enough. However we are not able to obtain such a pointwise estimate for $\partial_{t} \phi$. Rather we have to use the integral version of (2.16), namely

$$
e^{2 \phi}\left(1+|p|^{2}\right)=e^{2 \phi_{0}(X(0))}\left(1+|P(0)|^{2}\right)+2 \int_{0}^{t} e^{2 \phi} \partial_{s} \phi(s, X(s)) d s .
$$

Eventually the quantity we shall estimate is the time integral in the right hand side of (2.17). For this purpose we need the integral representation formula for $\partial_{t} \phi$ which was derived in $[6]$. We recall it here for the sake of reference:

$$
\partial_{t} \phi(t, x)=\left(\partial_{t} \phi\right)_{D}+\mathrm{I}+\mathrm{II}+\mathrm{III}
$$

where

$$
\begin{gathered}
\left(\partial_{t} \phi\right)_{D}=\partial_{t} \phi_{\text {hom }}-\frac{1}{t} \int_{|x-y|=t} \int \frac{f_{0}(y, p)}{(1+\omega \cdot \widehat{p})} \frac{d p}{\sqrt{1+|p|^{2}}} d S_{y} \\
\mathrm{I}=\int_{|x-y| \leqslant t} \int \frac{(\omega+\widehat{p}) \cdot \widehat{p}}{(1+\omega \cdot \widehat{p})^{2}} f(t-|x-y|, y, p) \frac{d p}{\sqrt{1+|p|^{2}}} \frac{d y}{|x-y|^{2}}, \\
\mathrm{II}=-\int_{|x-y| \leqslant t} \int \frac{(\omega+\widehat{p})^{2}}{(1+\omega \cdot \widehat{p})^{2}}(S \phi) f(t-|x-y|, y, p) \frac{d p}{\sqrt{1+|p|^{2}}} \frac{d y}{|x-y|}, \\
\mathrm{III}=-\int_{|x-y| \leqslant t} \int \frac{d p}{(\omega+\widehat{p}) \cdot \nabla_{x} \phi} f(t-|x-y|, y, p) \frac{d y}{\left(1+|p|^{2}\right)^{3 / 2}} \frac{d x-y \mid}{\mid x)^{2}}
\end{gathered}
$$

We rewrite the above representation formula in a new form which is suitable for being estimated in terms of the null cone energy:

Proposition 1 The representation formula (2.18) can be rewritten in the form

$$
\partial_{t} \phi(t, x)=\left(\partial_{t} \phi\right)_{D}+\sum_{i=0}^{5} \mathcal{Z}_{i}
$$

where

$$
\mathcal{Z}_{0}=-2 \int_{|x-y| \leqslant t}\left(\partial_{t} \phi\right) \mu(t-|x-y|, y) \frac{d y}{|x-y|}
$$




$$
\begin{gathered}
\mathcal{Z}_{1}=\int_{|x-y| \leqslant t} \int \frac{f(t-|x-y|, y, p)}{\sqrt{1+|p|^{2}}(1+\omega \cdot \widehat{p})} d p \frac{d y}{|x-y|^{2}}, \\
\mathcal{Z}_{2}=-\int_{|x-y| \leqslant t} \int \frac{f(t-|x-y|, y, p)}{\left(1+|p|^{2}\right)^{3 / 2}(1+\omega \cdot \widehat{p})^{2}} d p \frac{d y}{|x-y|^{2}}, \\
\mathcal{Z}_{3}=2 \int_{|x-y| \leqslant t}\left(\partial_{t} \phi-\omega \cdot \nabla_{x} \phi\right) \int \frac{(\omega \cdot \widehat{p}) f(t-|x-y|, y, p)}{\sqrt{1+|p|^{2}}(1+\omega \cdot \widehat{p})} d p \frac{d y}{|x-y|}, \\
\mathcal{Z}_{4}=\int_{|x-y| \leqslant t}\left(\partial_{t} \phi-\omega \cdot \nabla_{x} \phi\right) \int \frac{f(t-|x-y|, y, p)}{\left(1+|p|^{2}\right)^{3 / 2}(1+\omega \cdot \widehat{p})^{2}} d p \frac{d y}{|x-y|}, \\
\mathcal{Z}_{5}=-2 \int_{|x-y| \leqslant t}\left(\omega \wedge \nabla_{x} \phi\right) \cdot \int \frac{(\omega \wedge \widehat{p}) f(t-|x-y|, y, p)}{\sqrt{1+|p|^{2}}(1+\omega \cdot \widehat{p})} d p \frac{d y}{|x-y|} .
\end{gathered}
$$

Proof: This proposition is the result of a straightforward calculation which proceeds as follows. In the integral I of (2.18) we write

$$
(\omega+\widehat{p}) \cdot \widehat{p}=(1+\omega \cdot \widehat{p})-\left(1+|p|^{2}\right)^{-1},
$$

which shows that I $=\mathcal{Z}_{1}+\mathcal{Z}_{2}$. In the integrals II and III of (2.18) we decompose $\nabla_{x} \phi$ into a component parallel to $\omega$ and a component orthogonal to $\omega$, i.e., we write

$$
\nabla_{x} \phi=\left(\omega \cdot \nabla_{x} \phi\right) \omega-\omega \wedge \omega \wedge \nabla_{x} \phi .
$$

It follows that

$$
S \phi=\left(\partial_{t} \phi\right)(1+\omega \cdot \widehat{p})-\left(\partial_{t} \phi-\omega \cdot \nabla_{x} \phi\right)(\omega \cdot \widehat{p})+\left(\omega \wedge \nabla_{x} \phi\right) \cdot(\omega \wedge \widehat{p})
$$

and

$$
(\omega+\widehat{p}) \cdot \nabla_{x} \phi=\left(\omega \cdot \nabla_{x} \phi\right)(1+\omega \cdot \widehat{p})+\left(\omega \wedge \nabla_{x} \phi\right) \cdot(\omega \wedge \widehat{p}) .
$$

In the integral II we also use

$$
(\omega+\widehat{p})^{2}=2(1+\omega \cdot \widehat{p})-\left(1+|p|^{2}\right)^{-1} .
$$

After substituting and summing up the various terms one can easily verify that

$$
\mathrm{II}+\mathrm{III}=\mathcal{Z}_{0}+\mathcal{Z}_{3}+\mathcal{Z}_{4}+\mathcal{Z}_{5}
$$

which concludes the proof.

We conclude this section with

Lemma 2 For $R>1, a, b \geqslant 0$, denote

$$
\mathcal{B}_{a b}(R)=\int_{|p| \leqslant R}\left(\sqrt{1+|p|^{2}}+\omega \cdot p\right)^{-a}\left(1+|p|^{2}\right)^{-b} d p .
$$

Then

$$
\begin{aligned}
& \mathcal{B}_{a, b}(R) \leqslant C R^{(2-2 b)} \log R, \quad \text { if } a=1, b<1 ; \\
& \mathcal{B}_{a, b}(R) \leqslant C R^{(3-2 b-a)}, \quad \text { if } a<1, b<\frac{3-a}{2} ; \\
& \mathcal{B}_{a, b}(R) \leqslant C R^{(1+a-2 b)}, \quad \text { if } a>1, b<\frac{1+a}{2} .
\end{aligned}
$$

Proof: The proof is by evaluating the integral in polar coordinates. 


\section{Proof of the main theorem}

Without loss of generality, we can assume $\mathcal{P}(t) \geqslant C$, where $C$ can be chosen arbitrarily large, otherwise we redefine $\mathcal{P}(t) \rightarrow \mathcal{P}(t)+C$. A first pointwise estimate on $\partial_{t} \phi$ follows by the results of the previous section.

\section{Proposition 2}

$$
\left|\partial_{t} \phi(t, x)\right| \leqslant 2 \int_{|x-y| \leqslant t}\left|\partial_{t} \phi\right| \mu(t-|x-y|, y) \frac{d y}{|x-y|}+C(t) \mathcal{P}(t)^{2} \log \mathcal{P}(t) .
$$

Proof: From Proposition [1 we have

$$
\left|\partial_{t} \phi(t, x)\right| \leqslant C(t)+2 \int_{|x-y| \leqslant t}\left|\partial_{t} \phi\right| \mu(t-|x-y|, y) \frac{d y}{|x-y|}+\sum_{i=1}^{5}\left|\mathcal{Z}_{i}\right| .
$$

Let us estimate each integral $\mathcal{Z}_{i}$, for $i=1, \ldots 5$. Observe that the domain of integration in the variable $p$ can be chosen as $\left\{|p| \leqslant 1+e^{-\phi} \mathcal{P}(t)\right\}$ by the definition of $\mathcal{P}(t)$.

Estimate for $\mathcal{Z}_{1}$ : By (2.12)-(2.14) and Lemma 2

$$
\begin{aligned}
\left|\mathcal{Z}_{1}\right| & \leqslant C \int_{|x-y| \leqslant t} e^{4 \phi} \mathcal{B}_{1,0}\left(1+e^{-\phi} \mathcal{P}(t)\right)(t-|x-y|, y) \frac{d y}{|x-y|^{2}} \\
& \leqslant C(t) \mathcal{P}(t)^{2} \int_{|x-y| \leqslant t} e^{2 \phi} \log \left(1+e^{-\phi} \mathcal{P}(t)\right)(t-|x-y|, y) \frac{d y}{|x-y|^{2}} \\
& \leqslant C(t) \mathcal{P}(t)^{2} \int_{|x-y| \leqslant t}\left(e^{2 \phi}|\phi|(t-|x-y|, y)+\log \mathcal{P}(t)\right) \frac{d y}{|x-y|^{2}} \\
& \leqslant C(t) \mathcal{P}(t)^{2} \log \mathcal{P}(t) .
\end{aligned}
$$

Estimate for $\mathcal{Z}_{2}$ : Again by (2.12)-(2.14) and Lemma 2,

$$
\begin{aligned}
\left|\mathcal{Z}_{2}\right| & \leqslant C \int_{|x-y| \leqslant t} e^{4 \phi} \mathcal{B}_{2,1 / 2}\left(1+e^{-\phi} \mathcal{P}(t)\right)(t-|x-y|, y) \frac{d y}{|x-y|^{2}} \\
& \leqslant C(t) \mathcal{P}(t)^{2} \int_{|x-y| \leqslant t} e^{2 \phi(t-|x-y|, y)} \frac{d y}{|x-y|^{2}} \leqslant C(t) \mathcal{P}(t)^{2} .
\end{aligned}
$$

Estimate for $\mathcal{Z}_{3}$ : By the Cauchy-Schwarz inequality, (1.9), (2.12)-(2.14) and Lemma 2

$$
\begin{aligned}
\left|\mathcal{Z}_{3}\right| \leqslant & C \int_{|x-y| \leqslant t}\left|\partial_{t} \phi-\omega \cdot \nabla_{x} \phi\right| e^{4 \phi} \mathcal{B}_{1,0}\left(1+e^{-\phi} \mathcal{P}(t)\right)(t-|x-y|, y) \frac{d y}{|x-y|} \\
\leqslant & C\left(\int_{|x-y| \leqslant t}\left|\partial_{t} \phi-\omega \cdot \nabla_{x} \phi\right|^{2}(t-|x-y|, y)\right)^{1 / 2} \\
& \times\left(\int_{|x-y| \leqslant t} e^{8 \phi}\left[\mathcal{B}_{1,0}\left(1+e^{-\phi} \mathcal{P}(t)\right)\right]^{2}(t-|x-y|, y) \frac{d y}{|x-y|^{2}}\right)^{1 / 2} \\
\leqslant & C(t) \mathcal{P}(t)^{2} \log \mathcal{P}(t) .
\end{aligned}
$$


Estimate for $\mathcal{Z}_{4}$ : As before,

$$
\begin{aligned}
\left|\mathcal{Z}_{4}\right| \leqslant C & \left(\int_{|x-y| \leqslant t}\left|\partial_{t} \phi-\omega \cdot \nabla_{x} \phi\right|^{2}(t-|x-y|, y) d y\right)^{1 / 2} \\
& \quad \times\left(\int_{|x-y| \leqslant t} e^{8 \phi}\left[\mathcal{B}_{2,1 / 2}\left(1+e^{-\phi} \mathcal{P}(t)\right)\right]^{2}(t-|x-y|, y) \frac{d y}{|x-y|^{2}}\right)^{1 / 2} \\
\leqslant & \leqslant C(t) \mathcal{P}(t)^{2} .
\end{aligned}
$$

Estimate for $\mathcal{Z}_{5}$ : Again as before,

$$
\begin{aligned}
\left|\mathcal{Z}_{5}\right| \leqslant & C\left(\int_{|x-y| \leqslant t}\left|\omega \wedge \nabla_{x} \phi\right|^{2}(t-|x-y|, y, p) d y\right)^{1 / 2} \\
& \times\left(\int_{|x-y| \leqslant t} e^{8 \phi}\left[\mathcal{B}_{1,0}\left(1+e^{-\phi} \mathcal{P}(t)\right)\right]^{2}(t-|x-y|, y) \frac{d y}{|x-y|^{2}}\right)^{1 / 2} \\
\leqslant & C(t) \mathcal{P}(t)^{2} \log \mathcal{P}(t) .
\end{aligned}
$$

Replacing the preceding estimates in (3.19) concludes the proof.

Using Proposition 2 and (2.17) we obtain the integral inequality

$$
\begin{aligned}
e^{2 \phi}\left(1+|p|^{2}\right) & \leqslant C+2 \int_{0}^{t} e^{2 \phi}\left|\partial_{s} \phi(s, X(s))\right| d s \\
& \leqslant C+C(t) \int_{0}^{t} \mathcal{P}(s)^{2} \log \mathcal{P}(s) d s+4 I_{0}\left(\left|\partial_{t} \phi\right| \mu, t\right)
\end{aligned}
$$

where

$$
I_{0}(g, t)=\int_{0}^{t} e^{2 \phi(s, X(s))} \int_{|X(s)-y| \leqslant s} g(s-|X(s)-y|, y) \frac{d y}{|X(s)-y|} d s .
$$

We rewrite $I_{0}(g, t)$ as

$$
I_{0}(g, t)=\int_{0}^{t} \mathcal{I}_{0}(g, \tau, t) d \tau
$$

where

$$
\mathcal{I}_{0}(g, \tau, t)=\int_{\tau}^{t} e^{2 \phi(s, X(s))} \int_{|y|=s-\tau} \frac{g(\tau, X(s)-y)}{(s-\tau)} d S_{y} d s .
$$

Except for the factor $e^{2 \phi(s, X(s))}, \mathcal{I}_{0}(g, \tau, t)$ is the integral which is estimated in the proof of [17. Lemma 1.1]. However, since we shall need a slightly different formulation of the estimate proved in [17, we present here a complete proof of the result that we are going to use:

Lemma 3 For all $0 \leqslant \tau \leqslant t$,

$$
\mathcal{I}_{0}(g, \tau, t) \leqslant C(t) \frac{\|g(\tau)\|_{L^{2}}}{\sqrt{t-\tau}} \int_{\tau}^{t} \log \mathcal{P}(s) d s
$$


Proof: We first rewrite $\mathcal{I}_{0}$ in spherical coordinates:

$$
\mathcal{I}_{0}(g, \tau, t)=\int_{\tau}^{t} e^{2 \phi(s, X(s))} \int_{0}^{\pi} \int_{0}^{2 \pi} g(\tau, X(s)-(s-\tau) \omega)(s-\tau) \sin \theta d \varphi d \theta d s,
$$

where $\omega=\omega(\theta, \varphi)=(\sin \theta \cos \varphi, \sin \theta \sin \phi, \cos \theta)$. Now, in [17, Lemma 2.1] it is shown that the transformation of variables $(s, \theta, \varphi) \rightarrow X(s)-(s-\tau) \omega$ is a $C^{1}$ diffeomorphism with Jacobian

$$
J=(\dot{X}(s) \cdot \omega-1)(s-\tau)^{2} \sin \theta=(\widehat{P}(s) \cdot \omega-1)(s-\tau)^{2} \sin \theta .
$$

Hence, applying Cauchy-Schwarz's inequality,

$$
\begin{aligned}
\mathcal{I}_{0}(g, \tau, t) \leqslant & \left(\int_{\tau}^{t} \int_{0}^{\pi} \int_{0}^{2 \pi} g^{2}(\tau, X(s)-(s-\tau) \omega)|J| d \varphi d \theta d s\right)^{1 / 2} \\
& \times\left(\int_{\tau}^{t} e^{4 \phi(s, X(s))} \int_{0}^{\pi} \int_{0}^{2 \pi} \frac{\sin \theta}{(1-\widehat{P}(s) \cdot \omega)} d \varphi d \theta d s\right)^{1 / 2} \\
\leqslant & \|g(\tau)\|_{L^{2}}\left(\int_{\tau}^{t} e^{4 \phi(s, X(s))} \int_{0}^{\pi} \int_{0}^{2 \pi} \frac{\sin \theta}{(1-\widehat{P}(s) \cdot \omega)} d \varphi d \theta d s\right)^{1 / 2} .
\end{aligned}
$$

We estimate the angular integral as

$$
\begin{aligned}
\int_{0}^{\pi} \int_{0}^{2 \pi} \frac{\sin \theta}{(1-\widehat{P}(s) \cdot \omega)} d \varphi d \theta d s & =2 \pi \int_{-1}^{1} \frac{d u}{(1-|\widehat{P}(s)| u)} \\
& \leqslant C(1-\log (1-|\widehat{P}(s)|)) \leqslant C(|\phi|+\log \mathcal{P}(s)) .
\end{aligned}
$$

We finally obtain

$$
\begin{aligned}
\mathcal{I}_{0}(g, \tau, t) & \leqslant C(t)\|g(\tau)\|_{L^{2}}\left(\int_{\tau}^{t}\left(e^{\phi}|\phi|+\log \mathcal{P}(s)\right) d s\right)^{1 / 2} \\
& \leqslant C(t) \frac{\|g(\tau)\|_{L^{2}}}{\sqrt{t-\tau}} \int_{\tau}^{t} \log \mathcal{P}(s) d s
\end{aligned}
$$

which concludes the proof of the lemma.

The proof of Theorem 1 is now almost complete. Observe that, by (2.15) and (1.8),

$$
\left\|\partial_{t} \phi \mu(\tau)\right\|_{L^{2}} \leqslant\|\mu(\tau)\|_{\infty}\left\|\partial_{t} \phi(\tau)\right\|_{L^{2}} \leqslant C(t) \mathcal{P}(\tau)^{2}, \quad \tau \leqslant t .
$$

Thus

$$
\mathcal{I}_{0}\left(\left|\partial_{t} \phi\right| \mu, \tau, t\right) \leqslant C(t) \frac{\mathcal{P}(\tau)^{2}}{\sqrt{t-\tau}} \int_{\tau}^{t} \log \mathcal{P}(s) d s
$$


Hence the integral $I_{0}\left(\left|\partial_{t} \phi\right| \mu, t\right)$ is bounded by

$$
\begin{aligned}
I_{0}\left(\left|\partial_{t} \phi\right| \mu, t\right) & \leqslant C(t) \int_{0}^{t} \int_{\tau}^{t} \frac{\mathcal{P}(\tau)^{2}}{\sqrt{t-\tau}} \log \mathcal{P}(s) d s d \tau \\
& =C(t) \int_{0}^{t} \int_{0}^{s} \frac{\mathcal{P}(\tau)^{2}}{\sqrt{t-\tau}} \log \mathcal{P}(s) d \tau d s \\
& \leqslant C(t) \int_{0}^{t} \mathcal{P}(s)^{2} \log \mathcal{P}(s) d s .
\end{aligned}
$$

Finally, going back to (3.20) we obtain the Grönwall inequality

$$
\mathcal{P}(t)^{2} \leqslant C(t)\left(1+\int_{0}^{t} \mathcal{P}(s)^{2} \log \mathcal{P}(s) d t\right),
$$

whence $\mathcal{P}(t) \leqslant C(t)$. By Lemma this completes the proof of the main theorem.

Acknowledgments: The author acknowledges support by the European HYKE network (contract HPRN-CT-2002-00282) and by the project "PDE and Harmonic Analysis", sponsored by Research Council of Norway (proj. no. 160192/V30).

\section{References}

[1] H. Andréasson: The Einstein-Vlasov System/Kinetic Theory. Living Rev. Relativity 8 (cited on 7 July 2005)

[2] H. Andréasson, S. Calogero, G. Rein: Global classical solutions to the spherically symmetric Nordström-Vlasov system. Math. Proc. Camb. Phil. Soc. 138, 533-539 (2005)

[3] F. Bouchut, F. Golse, C. Pallard: Classical Solutions and the GlasseyStrauss Theorem for the 3D Vlasov-Maxwell System. Arch. Rat. Mech. Anal. 170, 1-15 (2003)

[4] S. Calogero: Spherically symmetric steady states of galactic dynamics in scalar gravity. Class. Quant. Gravity 20, 1729-1741 (2003)

[5] S. Calogero, H. Lee: The non relativistic limit of the Nordström-Vlasov system. Commun. Math. Sci. 2, 19-34 (2004)

[6] S. Calogero, G. Rein: On classical solutions of the Nordström-Vlasov system. Commun. Partial Diff. Eqns. 28, 1863-1885 (2003)

[7] S. Calogero, G. Rein: Global weak solutions to the Nordström-Vlasov system. J. Diff. Eqns. 204, 323-338 (2004)

[8] T. Damour, G. Esposito-Farese: Tensor-multi-scalar theories of gravitation. Class. Quantum Grav. 9, 2093-2176 (1992) 
[9] R. J. DiPerna, P. L. Lions: Global weak solutions of Vlasov-Maxwell systems. Commun. Pure Appl. Math. 52, 729-757 (1989)

[10] S. Friedrich: Global Small Solutions of the Vlasov-Nordström System. (preprint)

[11] R. Glassey, W. Strauss: Singularity formation in a collisionless plasma could occur only at high velocities. Arch. Rat. Mech. Anal. 92, 59-90 (1986)

[12] R. Glassey, W. Strauss: Absence of shocks in an initially dilute collisionless plasma. Commun. Math. Phys. 113, 191-208 (1987)

[13] R. Glassey, J. Schaeffer: The "Two and One-Half Dimensional" Relativistic Vlasov Maxwell System. Commun. Math. Phys. 185, 257-284 (1997)

[14] S. Klainerman, G. Staffilani: A new approach to study the Vlasov-Maxwell system. Commun. Pure Appl. Anal. 1, 103-125 (2002)

[15] H. Lee: Global existence of solutions of the Nordström-Vlasov system in two space dimensions. Commun. Partial Diff. Eqns. (to appear)

[16] P.-L. Lions, B. Perthame: Propagation of moments and regularity for the 3-dimensional Vlasov-Poisson system. Invent. Math. 105, 415-430 (1991)

[17] C. Pallard: On the boundness of the momentum support of solutions to the relativistic Vlasov-Maxwell system. Indiana Univ. Math. J. (to appear)

[18] K. Pfaffelmoser: Global classical solutions of the Vlasov-Poisson system in three dimensions for general initial data. J. Diff. Eqns. 95, 281-303 (1992)

[19] G. Rein: Generic global solutions of the relativistic Vlasov-Maxwell system of plasma physics. Commun. Math. Phys. 135, 41-78 (1990)

[20] G. Rein: Selfgravitating systems in Newtonian theory - the Vlasov-Poisson system. Banach Center Publications 41, Part I, 179-194 (1997)

[21] G. Rein, A. D. Rendall: Global existence of solutions of the spherically symmetric Vlasov-Einstein system with small initial data. Commun. Math. Phys. 150, 561-583 (1992)

[22] G. Rein, A. D. Rendall: The Newtonian limit of the Spherically Symmetric Vlasov-Einstein System. Commun. Math. Phys. 150, 585-591 (1992)

[23] A. D. Rendall: The Newtonian limit for asymptotically flat solutions of the Vlasov-Einstein system. Commun. Math. Phys. 163, 89-112 (1994)

[24] J. Schaeffer: Global existence of smooth solutions to the Vlasov-Poisson system in three dimensions. Commun. Part. Diff. Eqns. 16, 1313-1335 (1991)

[25] S. L. Shapiro, S. A. Teukolsky: Scalar gravitation: A laboratory for numerical relativity. Phys. Rev. D 47, 1529-1540 (1993) 